\title{
Supporting Locate and Search Operations Based on Drift Model and Bayesian Probability
}

\author{
Wei Lu*
}

School of Chemical Engineering, Anhui University of Science \& Technology, Huainan, China,+86-0554-6632810

\begin{abstract}
With the rapid development of international aviation, air disaster often happens. When the plane crashed in the vast ocean, how to locate the wreckage and fast and effective rescue is worth studying. This paper focuses on locating the crashed plane, determining the rescue area and the search strategies. To locate the crashed plane, the mechanics principle of the crashed plane is analyzed and the dynamic differential equation which can be solved by applying the fourth-order Runge-Kutta algorithm was built. Substituting the related parameters into the numerical solution, We can get the final trajectory and the velocity of the crashed plane. Thus, the crash site can be roughly determined. To determine of rescue, according to the ocean currents of the crash site and the suspicious area, the drifting trajectory of the main plane wreckage to set up rescue area can be roughly confirmed. For the search strategies, the Bayesian probability is applied in the search area. Firstly, the search area can be divided into several small areas which can be represented by appropriate search probabilities in term of the distances between among of drifting trajectories and other subjective information. The closer distance, the greater the priority search probability. Then the search work can be started a prior in the small area with the highest probability. If the crashed plane cannot be found, the priority search probability of every small areas should be updated and repeat the previous work until the end of the search.
\end{abstract}

Keywords: Air disaster, search strategies, drift model, bayesian probability, the dynamic differential equation, the fourth-order runge-kutta algorithm.

\section{INTRODUCTION}

In order to indicate the origin of "supporting locate and search operations based on drift model and bayesian probability" problems, the following introduction is worth mentioning. Search and rescue operations are often characterized by a similar set of constraints: time is critical and any delay can result in dramatic consequences potentially human losses; operational environments are unfriendly, e.g. disaster scenes, air disaster, etc. Some mathematics model have already demonstrated their benefit of seaching the Malaysia Airlines Flight 370 which was a scheduled international passenger flight which is disappeared on Saturday, 8 March 2014, while flying from Kuala Lumpur International Airport to Beijing Capital International Airport.

In this paper, our focus is to build a generic mathematical model in order to assist "searchers" to plan a useful search for a lost plane feared to have crashed in open water while flying from Point A to Point B. The analysis of locate and search strategies when the time to find the victim (also referred to as target) is the optimization criterion. In particular, It is assumed that there are no signals from the downed plane and there are many different types of planes for which the "searchers" might be searching.
The remainder of the paper is organized as follows. In Section 2, how to locate the crashed plane was described [1]. In Section 3, the drifting trajectory of the main plane wreckage in order to set up rescue area was confirmed to determine of rescue. In Section 4, for the search strategies, the Bayesian probability is applied in the search area. Finally, a conclusion is provided in Section 5.

Next, we describe the data and methodology used in more detail.

\section{THE MODEL FOR DETERMINING THE LOCATION OF CRASHED PLANE}

It is considered that the plane has been exerted by gravity and airflow resistance with respect to air density. The air density is greatly changing within $10 \mathrm{~km}$ height. Therefore, the density is considered as the distribution of vertical direction. Firstly, we decompose gravity and airflow resistance to the tangential direction and normal direction of its movement, and obtain tangential acceleration and normal acceleration. Then we establish kinetic differential equations on plane coordinate system. Finally, we apply the fourthorder Runge-Kutta algorithm to get the solution of differential equation namely the track of crashed plane by using the software Maple [2].

In order to prevent the plane plummeted down to earth because of loss of speed, the driver would take the initiative to give up a part of the height of the plane to obtain enough speed after the breakdown of engine. That is, converting 
gravitational potential energy to kinetic energy. This operation is called unpowered glide.

After the loss of plane engine, according to the assumptions, the plane will land with headlong down the land, at this time. There are two forces act on the plane. $G$ is the gravity and is the wind forces, as shown in Fig. (1). In order to prevent the plane plummeted down to earth because of loss of speed, the driver would take the initiative to give up a part of the height of the plane to obtain enough speed after the breakdown of engine. That is, converting gravitational potential energy to kinetic energy. This operation is called unpowered glide.

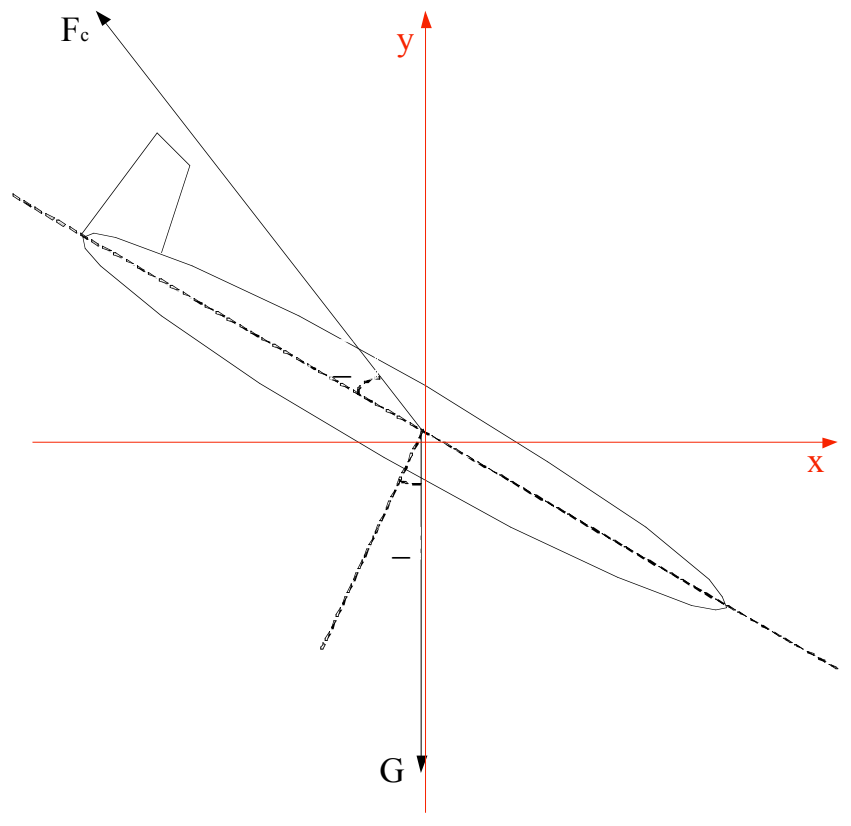

Fig.(1). The stress diagram for the plane wreckage.

The other two parameters is the angle $\theta$ (witch is between the horizon and the path of the down plane) and the angle $\alpha$ (which is between the $F_{C}$ and the path of the down plane), the angle $\alpha$ is got from the calculation of lift-to-drag ratio (when a plane flying, the ratio of lift and drag at the same angle). As shown in Fig. (1).

We can get the kinetic equation in the horizontal and vertical directions from the formula (1) and (2).

$M g \sin \theta-F_{C} \cos \theta=M \frac{d v}{d t}$

$M g \cos \theta-F_{C} \sin \theta=M v \frac{d \theta}{d t}$

It is observed that the relationship exists between angle and the component of velocity can be described as the formula (3), obviously, the component of velocity and the actual velocity satisfy the formula (4). $\tan (\pi-\theta)=\frac{d y}{d x}=\frac{\dot{x}}{\dot{y}}$

$V=\sqrt{\dot{x}^{2}+\dot{y}^{2}}$

The calculation formulas of $F_{c}$ can be

$F_{C}=\frac{1}{2} C_{D} \rho S v^{2}$

Where.

$C_{D}=0.12149-0.01714 C_{L}^{2}+0.00690 C_{L}^{3}$

The formulas for lift force can be

$L=\frac{1}{2} \rho V^{2} C_{L} S$

Where.

$C_{L}=1.1488+0.11832 \alpha^{2}+0.00092 \alpha^{3}$

\subsection{The Solution of the Model}

For the classical plane, the wing's reference area $S$ is $75 \mathrm{~m}^{2}$, plane weight is $20 t$, the initial values of $\theta$ is 0 , the flying velocity is $900 \mathrm{~km} / \mathrm{h}$ (about $275 \mathrm{~m} / \mathrm{s}$ ), We can ignore some flight attitudes of the plane which is located 10000 meters above the sea level. For general plane, the low-speed or the subsonic plane's Lift-to-drag ratio can reach $17 \sim 18$, then, let $\alpha=\arctan 1 / 17$. According to the established model and the requirements of the problem, the equations can be solved by using Runge-Kutta method.

Substituting the equation (5)-(8) into the original equation, we can obtain

$$
\begin{aligned}
& -g \frac{\dot{y}}{\sqrt{\dot{x}^{2}+\dot{y}^{2}}}-\frac{1}{2 M} \rho C S\left(\dot{x}^{2}+\dot{y}^{2}\right) \cos \alpha=\frac{\dddot{x} \dot{x}+\ddot{y} \ddot{y}}{\sqrt{\dot{x}^{2}+\dot{y}^{2}}} \\
& g \frac{\dot{x}}{\sqrt{\dot{x}^{2}+\dot{y}^{2}}}-\frac{1}{2 M} \rho C S\left(\dot{x}^{2}+\dot{y}^{2}\right) \sin \alpha=-\frac{\ddot{y} \dot{x}-\ddot{y} \ddot{x}}{\sqrt{\dot{x}^{2}+\dot{y}^{2}}}
\end{aligned}
$$

The higher-order singularly perturbed ordinary differential equations are reduced to the low-order differential equations in order to give numerical solution.

If:

$$
\begin{aligned}
& x_{1}=x \quad x_{3}=y \\
& x_{2}=\dot{x} \quad x_{4}=\dot{y}
\end{aligned}
$$

And then substituting the equation (11), (12) into equation (9) and (10), we get four first order differential equations as follows:

$\dot{x}_{1}=x_{2}$ 
$\dot{x}_{2}=-\frac{1}{2 M} \rho C S \sqrt{\dot{x}^{2}+\dot{y}^{2}}(\dot{\mathrm{y}} \sin \alpha+\dot{x} \cos \alpha)$

$\dot{x}_{3}=x_{4}$

$\dot{x}_{4}=-g+\frac{1}{2 M} \rho C S \sqrt{\dot{x}^{2}+\dot{y}^{2}}(\dot{x} \sin \alpha-\dot{y} \cos \alpha)$

$\rho$ is a variation with respect to the height, so we need to analysis and solve it with height changed. It can be considered that the weight of the air column is equal to its pressure in the numerical values, according to this thought to obtain the result.

$-d p=1.293 \times \frac{P}{101325} \times w \times 9.80665 \times d h$

In the equation (17): $1.293 \mathrm{~kg} / \mathrm{m}^{3}$ is the density under normal atmospheric conditions $\left(1 \mathrm{~atm}, 0{ }^{\circ} \mathrm{C}, 101.325 \mathrm{kP}\right) . P$ is the pressure's correction coefficient on the density, $w$ is the temperature's correction coefficient on the density, $\mathrm{g}=9.80665 \mathrm{~m} / \mathrm{s}^{2}, h$ is altitude , $p$ is the air pressure at $h$.

Set temperature of sea-level as $15^{\circ} \mathrm{C}$ and the temperature of the altitude of $10 \mathrm{~km}$ as $50^{\circ} \mathrm{C}$. The temperature variation is homogeneous and air density is inversely proportional to the absolute temperature, thus

$$
w=\frac{273.15}{253.15+15-\frac{15+50}{10000} \cdot h}
$$

Substituting the equation (18) into the equation (17) and then doing a integral, we put the special values conditions into formula to get the final result.

$P=e^{5.25885 \ln (288.15-0.006 \mathrm{~h})-18.25731}$

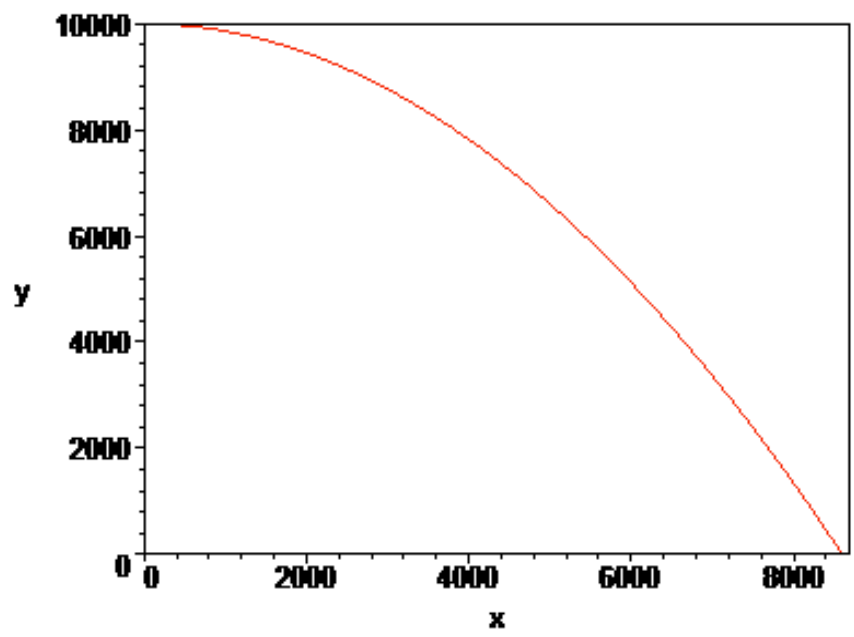

Fig. (2). The trajectory of the plane from a height of $10 k$ meters.
As shown in Fig. (2), the distance is about $8.6 k$ meters from the site. The lateral velocity and the longitudinal velocity of the plane when it crashed into the sea from different heights can be calculated by maple programming.

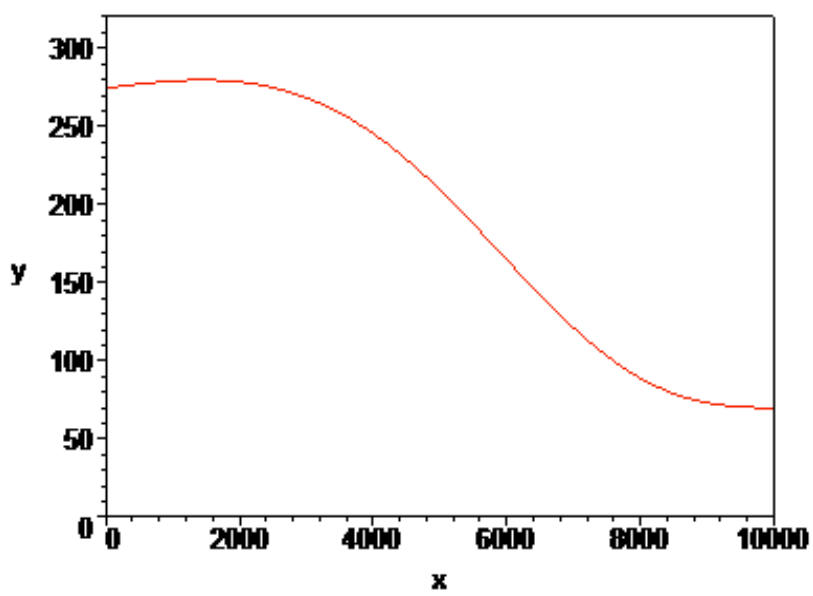

Fig. (3). The horizontal velocity of the plane from a height of $10 k$ meters.

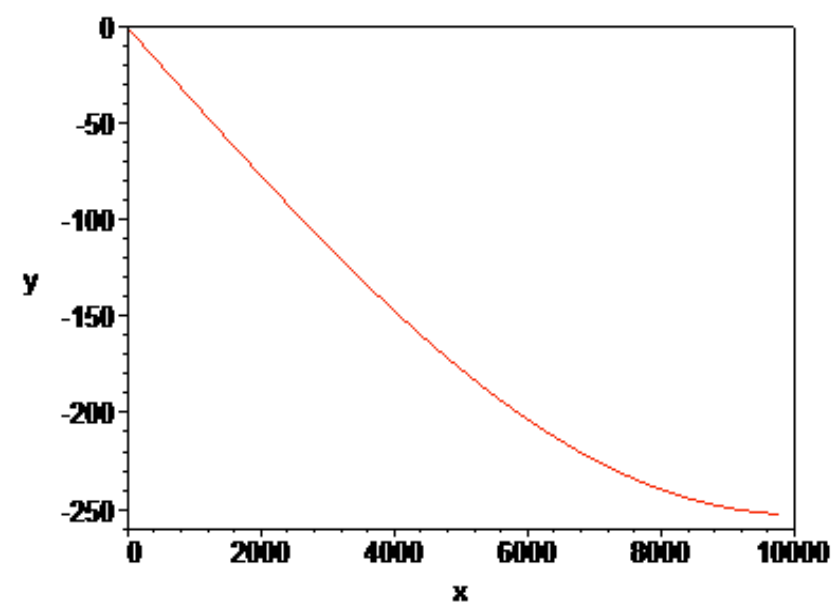

Fig. (4). The vertical velocity of the plane from a height of $10 k$ meters.

The horizontal and vertical velocity of the plane which crashed into the sea from a height of $10 k$ meters is simulated. The plane crashed into the sea level at the horizontal velocity of $70 \mathrm{~m} / \mathrm{s}$ and the vertical velocity is $-250 \mathrm{~m} / \mathrm{s}$. As shown in Figs. (3 and 4), the left y-axis is height $(m), \mathrm{x}$-axis is the velocity $(\mathrm{m} / \mathrm{s})$ of the plane, the right $\mathrm{y}$-axis is height $(m)$, the $\mathrm{x}$-axis is the vertical velocity $(\mathrm{m} / \mathrm{s})$ of the plane.

\subsection{The Determination of Suspicious Area based on Drift Model}

Carried by sea water and pushed by wind force, the plane wreckages floating on the sea surface drifts forward and 
deviate the original position [3]. Set the original position of the object as $\vec{S}_{0}$, through the time step of $\Delta t$, it drifts to another position which is set as $\vec{S}$, then it can be

$\vec{S}=\vec{S}_{0}+\int_{t_{0}}^{t_{0}+\Delta t} \overrightarrow{V_{t}} d t$

$\vec{V}$ stands for the plane wreckages' velocity at time $t$ and it is a function related to space and time. With this velocity, the plane wreckages eventually moves to $\vec{S}$ through the time step of $\Delta t$.the formula above is called as The EulerLagrange tracing method. If the $\Delta t$ is small enough, then we can take the velocity at $t_{0}$ as the velocity the plane wreckages owns in the period of $\Delta t$. By this way, when a tiny time step is set in the computer system, the motion trace of the plane wreckages then can be divided into a sequence of addings of discrete uniform motion. After it comes to $\vec{S}$,

The displacement in the next time step can be calculated out by using the velocity we have got. The main wreckage the offsetting model studied here is sea surface wind (ocean current).

Due to the lack of the detailed data of the Ocean's currents [5], it can't theoretically calculate the drifting trajectory of the plane wreckages, therefore, the following inference of the possible drifting trajectory of the wreckages is based on the hypothetical possible crash area and the general direction of the Southern Indian Ocean's currents.

Fig. (5) shows the possible crash area and the general direction of the Southern Indian Ocean's currents, and based on which it can infer the possible drifting trajectory and searching and salvage areas.

\section{THE SUSPICIOUS AREA SEARCH MODEL BASED ON BAYESIAN PROBABILITY}

As shown in Fig. (6), It is assumed that the probability of missing plane which is located in the area is $p$, the probability of successful search is $p$ in that area. If it is unable to find missing plane after searching here, the probability of successful search would update in this area to

$p^{\prime}=\frac{p(1-p)}{(1-p)+p(1-q)}=p \frac{1-q}{1-p q}<p$

For the other areas, if the probability of missing plane which is located in the other area is $r$, the probability is updated to

$$
r^{\prime}=r \frac{1}{1-p q}>r
$$

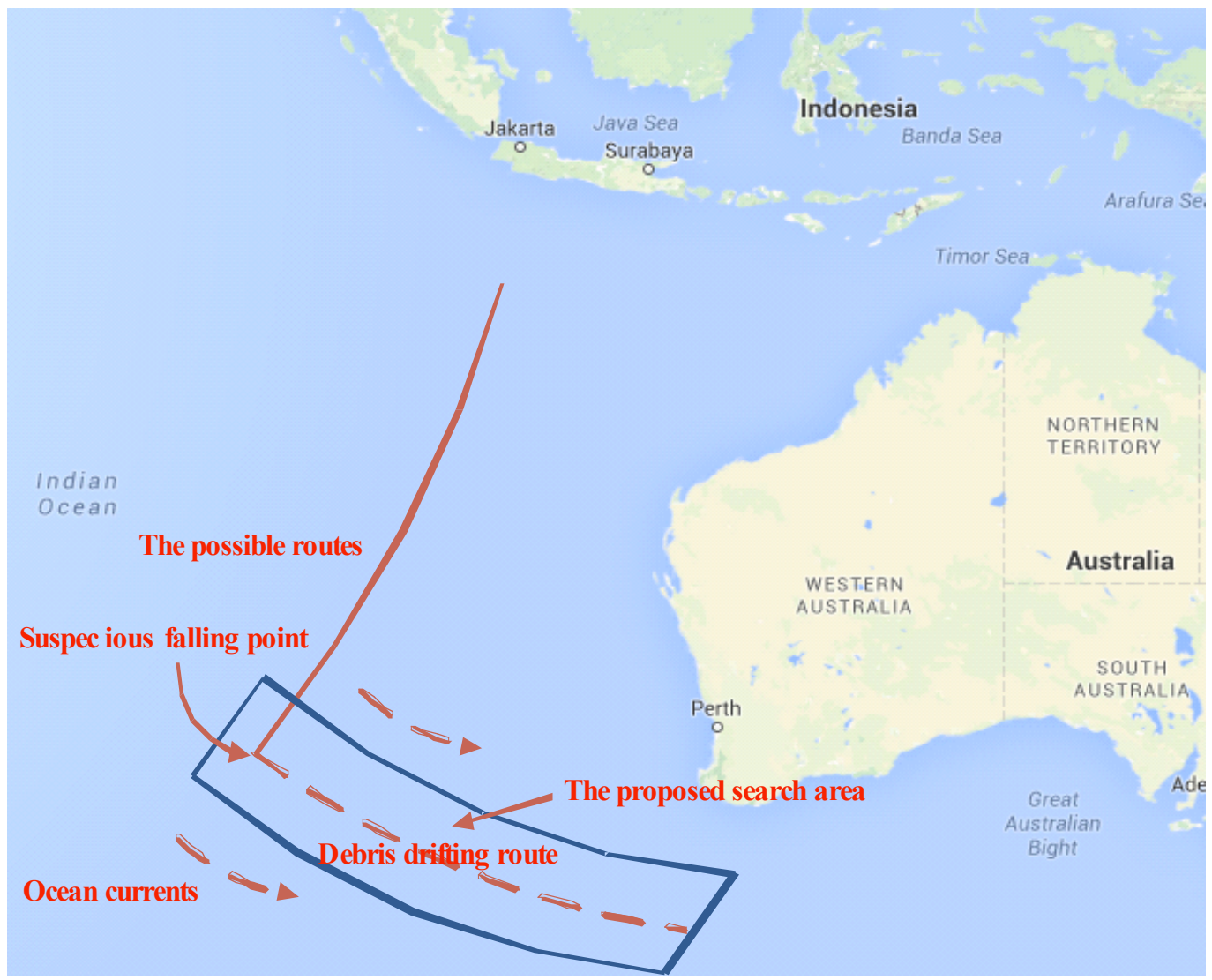

Fig. (5). The possible drifting trajectory and searching and salvage areas. 


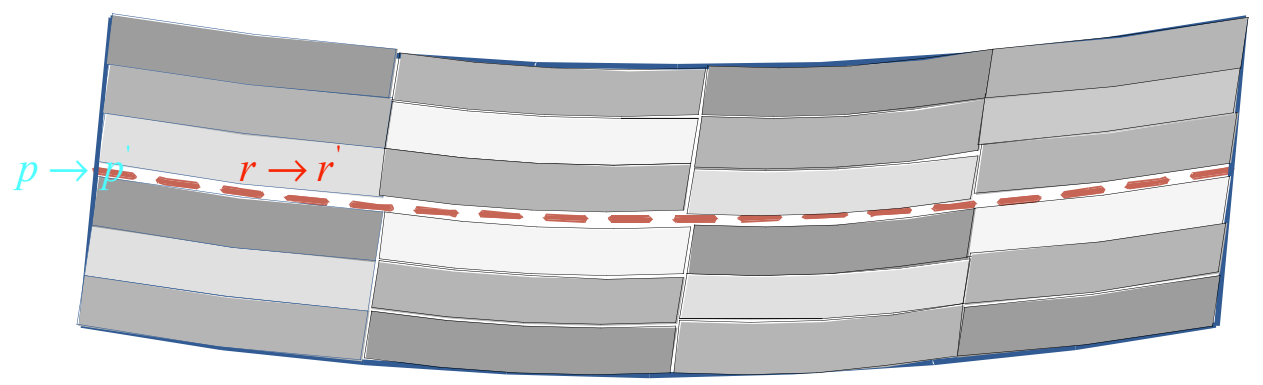

Fig. (6). The search probabilities in term of the distances between among of drifting trajectories and other subjective information.

For the search strategies, the Bayesian probability is applied in the search area. Firstly, the search area can be divided into several small areas which can be represented by appropriate search probabilities in term of the distances between among of drifting trajectories and other subjective information. The closer distance, the greater the priority search probability. Then the search work can be started a prior in the small area with the highest probability. If the crashed plane cannot be found, the priority search probability of every small areas should be updated and repeat the previous work until the end of the search [5-8].

As shown in Fig. (6), the deepest color represents the highest probability of missing plane which is located in this area. If the crashed plane cannot be found, the priority search probability of deepest color areas should be decreased while the search probability of other light color areas should be increased. Then, repeat the previous work until the end of the search $[9,10]$.

\section{CONCLUSION}

In this paper, we studied that the model which deduces the crash area based on rigorous principles of Physics. Its results of calculation is rather reliable; The searching strategy, which is based on the Bayes inference principle, is easier to be carried out. However, the inference of the plane wreckage's possible drifting trajectory and searching and salvage areas are only based on the crash area and the direction of the ocean currents, which is not rigorously calculated.

In future work, we intend to rigorously calculate the inference of the plane wreckage's possible drifting trajectory and searching and salvage areas by considering the complex directions of the ocean currents. We also intend to study how search algorithms based on drift model and bayesian probability when the number of states increases.

\section{CONFLICT OF INTEREST}

The author confirms that this article content has no conflicts of interest.

\section{ACKNOWLEDGEMENTS}

Declared none.

\section{REFERENCES}

[1] B. Jiang, "Research on crashed plane crashed down the sea and the trajectory of the black box", China High Technology Enterprises, vol. 20, pp: 2-3, 2014.

[2] P.G. Daniel, F.C. Jan, Y. Landau and E. Loiseau, "Drift modeling of cargo containers", Spill Science and Technology Bulletin, vol. 7, no. 5-6, pp:279-288, 2002.

[3] F.J.M. Davidson, A. Allen, G.B. Brassington, Ø. Breivik, P. Daniel, M. Kamachi, S. Sato, B. King, F Lefevre, M. Sutton and H. Kaneko, "Applications of GODAE ocean current forecasts to search and rescue and ship routing", Oceanography, vol. 22, no. 3, pp:176-181, 2009.

[4] M.S. Eide, Ø. Endresen, Ø. Breivik, O. W. Brude, I.H. Ellingsen, K. Røang, J. Hauge and P.O. Brett, "Prevention of Oil spill from shipping by modelling of dynamic risk", Marine Pollution Bulletin, vol. 54, no. 10, pp:1619-1633, 2007.

[5] B. Hackett, Ø. Breivik and C. Wettre, "Forecasting the drift of objects and substances in the oceans", In: Ocean Weather Forecasting: An Integrated View of Oceanography, E.P. Chassignet and J. Verron (Eds), pp:507-524, 2006.

[6] J.O.Berger, Statistical decision theory and bayesian analysis, Springer-Verlag, pp: 23-24, 2004.

[7] O. Yang, Research on the Ship out of Control Based on Drift Model, pp: 1-2, March 2008.

[9] P.L. Richardson, "Worldwide ship drift distributions identify missing data", Journal of Geophysics Research, vol. 94, no. C5, pp. 6169-6176, 1989.

[10] T. Suzuki, and H. Sato, "Measurement of the drifting of a fishing boat or research vessel due to wind and wave", The Journal of Japan's Institute of Navigation, vol. 57, pp:71-76, 1977.

[11] D.S. Massey, J. Arango, G. Hugo, A. Kouaouci, A. Pellegrino and J.E. Taylor, "An evaluation of international migration theory: The North American case", Population and Development Review, vol. 20, pp. 699-751, 1994. 